\title{
Trapping and Detection of Ions Generated in a High Magnetic Field Electrospray Ionization Fourier Transform Ion Cyclotron Resonance Mass Spectrometer
}

\author{
Steven A. Hofstadler and David A. Laude Jr. \\ Department of Chemistry and Biochemistry, University of Texas at Austin, Austin, Texas, USA
}

\begin{abstract}
The trapping and detection parameters employed with a Fourier transform ion cyclotron resonance (FTICR) mass spectrometer that is interfaced to a high magnetic field electrospray ionization (ESI) source are presented. ESI occurs at atmospheric pressure in a 1.5-T field, and FTICR detection occurs $25 \mathrm{~cm}$ away at $3.0 \mathrm{~T}$ in either one of two cells separated by a conductance limit and maintained at pressure differentials of $5 \times 10^{-5}$ and $2 \times 10^{-7}$ torr, respectively. The continuous electrospray ion current traversing the high- and low-pressure cells is 350 and $100 \mathrm{pA}$, respectively. Retarding grid studies at the high-pressure cell indicate electrospray ion kinetic energies are controllable from less than an electronvolt to more than $10 \mathrm{eV}$. These kinetic energies are a function of desolvating capillary-skimmer assembly distance and the skimmer potential. Efficient accumulation of injected ions is accomplished only when the trap-plate potential matches the ion kinetic energy. If this condition is satisfied, the trapped ion cell fills to the ion space charge limit within a few hundred milliseconds. It is concluded that even at the high pressures used, the primary trapping mechanism cannot be solely collision dependent because the rate of ion accumulation is independent of background pressure. However, optimized FTICR excitation conditions for peptides and proteins in the mass range from $10^{3}$ to more than $10^{6} \mathrm{kDa}$ are found to vary strongly with pressure; this is attributed to large mass- and charge-dependent differences in ion-molecule collision frequency. (J Am Soc Mass Spectrom 1992, 3, 615-623)
\end{abstract}

$\mathrm{E}$ lectrospray ionization (ESI) mass spectrometry enjoys increasing popularity today for high mass analysis of proteins and oligonucleotides $[1,2]$. This is the consequence of an ionization process that generates multiply charged ions and, independent of the size of molecules ranging to hundreds of kilodaltons, yields an analytical signal in the $\mathrm{m} / z \mathbf{5 0 0}$ to 2000 range that is accessible to most mass analyzers. ESI spectra were observed for proteins to about $200 \mathrm{kDa}$ [3] but the ionization source is capable of much higher performance, with ion currents generated for proteins beyond $500 \mathrm{kDa}$ [4] and for synthetic polymers beyond $1000 \mathrm{kDa}$ [5]. Unfortunately, the mass analyzers used to detect these ions were incapable of resolving the charge states to assign molecular weight values. In effect, the present restriction to ESI/MS high-mass analysis is not one of limited mass analyzer range, but one of limited mass analyzer resolution.

Consequently, the impetus for coupling double-

Address reprint requests to David A. Laude, Jr., Department of Chemistry and Biochemistry, University of Texas at Austin, Austin, TX 78712. sector and Fourier transform ion cyclotron resonance (FTICR) mass spectrometers to ESI is to achieve sufficient mass resolution and mass accuracy to resolve ESI isotopes within charge states. Promising results have been demonstrated with sector instruments [6-8]. For example. Bateman et al. [9] have recently achieved resolving power of 8500 ( $10 \%$ valley definition) for the $[\mathrm{M}+10 \mathrm{H}]^{10+}$ peak of bovine ubiquitin (MW = 8564.86). Low part-per-million mass measurement error was also obtained in the mass assignment of a number of proteins. The disadvantages of sector instruments for high resolution and high mass accuracy ESI analysis include compromised ion throughput, unintentional collision-induced dissociation due to the high kinetic energies employed, and susceptibility to corona discharge and arcing in the source [10].

FTICR [11-13] has recently been demonstrated as an attractive alternative to sector instruments for high resolution ESI/MS [14-17]. Compelling arguments for the coupling of ESI and FTICR are potentially superior mass resolution, mass accuracy, and sensitivity in the mass range of interest. For example, initial efforts by Henry and McLafferty et al. [14-16] yielded ESI/FTICR 
spectra of equine cytochrome $c(\mathrm{MW}=12,358.34)$ with a resolving power of 62,000 and chicken cytochrome $c$ (MW $=12,236.22)$ with a mass accuracy of $\pm 0.48 \mathrm{Da}$ [16]. However, sensitivity problems limited the general utility of their instrument because whereas most electrospray sources deliver 20 to $50 \mathrm{pA}$ currents to the mass analyzer [18], the external source FTICR was operated with no more than a few picoamperes measured at the cell. The problem is apparently that, as with other external source FTICR instruments $[19,20]$, ionization occurs more than a meter from the trapped ion cell and numerous electrostatic and/or quadrupole lenses are necessary to guide an initially broadly dispersed ion beam across a large magnetic field gradient to the trapped ion cell. Consequently, Henry and McLafferty et al. [15] observed poor spectral signal-tonoise ratio $(S / N)$ for larger proteins.

Recently we adopted a new approach to external source FTICR with the initial goal of increasing sensitivity in the ESI/FTICR experiment [17]. In this new design the electrospray ion source is positioned in the magnet solenoid used for FTICR detection with the hope that the strong confining nature of the magnetic field would direct the radial velocity component of the spray into small cyclotron orbits. In principle, ions would reach the analyzer cell with unit efficiency, with a consequent increase in FTICR sensitivity. Preliminary results with this magnetic field focusing design were encouraging, as a continuous electrospray current measuring in excess of $350 \mathrm{pA}$ was delivered to the cell. The ESI/FTICR spectrum of bovine albumin dimer at 132,532 Da was obtained with excellent $\mathrm{S} / \mathrm{N}$. The magnetic field focusing interface apparently delivers about one order of magnitude more current to the mass analyzer than is typically achieved with electrostatic optics for quadrupole instruments and more than 100 times the ion current than was achieved with any previous FTICR interface $[14,21,22]$. The primary limitation of our current instrument is inadequate differential pumping, which requires that FTICR detection occur at $2 \times 10^{-7}$ torr in the analyzer trapped ion cell compared to low $10^{-9}$ torr pressure in the Henry and McLafferty instrument [16]. A new instrument under construction in our laboratory should offer at least an order of magnitude reduction in pressure, yet retain the superior sensitivity of the magnetic field focusing interface design.

To be discussed in the present work are optimization studies for ion injection and trapping, and ion excitation and detection in the magnetic field focusing ESI/FTICR. Specifically, insights gained into the necessary relationship between ion kinetic energy and trap potential for efficient trapping of electrospray ions will be presented. Characteristic aspects of detection parameters necessary to maximize FTICR signal magnitude for large and multiply charged ions also will be discussed. The opportunity to acquire FTICR spectra in either of two trapped ion cells maintained at pressures of $5 \times 10^{-5}$ torr and $2 \times 10^{-7}$ torr will assist in distin- guishing collisional influences on both trapping and detection.

\section{Experimental}

ESI/FTICR interface. The electrospray source was based on the design by Chait and co-workers [23] who used a heated desolvating capillary and no countercurrent gas flow. The source was interfaced in our instrument with components that constitute the Extrel FTMS-2000 mass spectrometer, including 3.0-T superconducting magnet, Nicolet 1280 computer, cell controller, high-power excitation amplifier, and differentially pumped cubic dual cell assembly [24]. Our primary requirement for the interface was to operate the electrospray source at atmospheric pressure in the bore of the superconducting magnet and acquire FTICR spectra in the homogeneous region of the magnetic field at pressures in the $10^{-8}$-torr regime. As is shown in Figure 1, this was to be accomplished with a network of concentric tubes of increasing diameter that terminated at conductance limits positioned along the ion beam path. Dimensions and pressures along each stage of differential pumping are included in Figure 1. Beginning from the analyzer chamber, an unmodified Nicolet dual cell assembly separated by a $2-\mathrm{mm}$ conductance limit is mounted in a 4.5 -in. stainless steel tube and differentially pumped by twin $700 \mathrm{~L} / \mathrm{s}$ diffusion pumps to pressures of $2 \times 10^{-7}$ torr and $5 \times$ $10^{-5}$ torr during electrospray operation. A 2.187-in. i.d. stainless steel tube that terminates at a $4.7-\mathrm{mm}$ conductance limit is inserted and centered in the 4.5-in. tube a distance of $2.0 \mathrm{~cm}$ from the first trapped ion cell; pumping the 2.187 -in. tube with an $1100 \mathrm{~L} / \mathrm{s}$ cryopump achieves a pressure in the $10^{-4}$-torr range during electrospray operation. A 1.375-in. i.d. stainless steel vacuum chamber terminates with a skimmer cone assembly at a distance of $1.3 \mathrm{~cm}$ from the $4.7-\mathrm{mm}$ conductance limit in the 2.187-in. tube. The copper skimmer cone has a 500- $\mu \mathrm{m}$ orifice and $25^{\circ}$ total internal angle at the orifice. The 1.375-in. tube is pumped by a 13-L/s rough pump to 3 torr during electrospray operation.

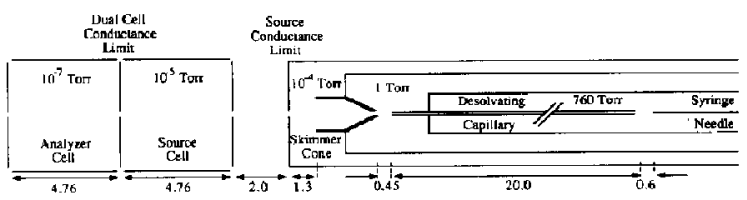

Figure 1. Electrode configuration and operating pressures for the high magnetic field electrospray source. All dimensions are in centimeters. The diameters of the source and dual cell conductance limits are 4.7 and $2.0 \mathrm{~mm}$, respectively, for the high-pressure experiment in which FTICR spectra are acquired in the source cell at $5 \times 10^{-5}$ torr. Conductance limit dimensions are reversed for the experiment in which ions are detected at $2 \times$ $10^{-7}$ torr in the analyzer cell. 
A 52 -in. long by 0.75 -in. diameter stainless steel probe serves as the housing for an electrospray interface that includes a $150-\mu \mathrm{m}$ i.d. blunt-ended electrospray syringe needle positioned $6 \mathrm{~mm}$ from a $500-\mu \mathrm{m}$ i.d. $\times 20-\mathrm{cm}$ desolvating capillary. The electrospray needle and desolvating capillary are mounted colinearly in a Delrin guide assembly; the area around the junction of the needle and desolvating capillary was machined away to create the electrospray cavity. The desolvating capillary, which is biased at $330 \mathrm{~V}$, protrudes several centimeters from the end of the probe housing to within $4.5 \mathrm{~mm}$ of the skimmer cone orifice. Overall, a distance of $29 \mathrm{~cm}$ separates the electrospray syringe needle operating at atmospheric pressure in a 1.5-T magnetic field from the analyzer cell operating at $2 \times 10^{-7}$ torr in a 3.0-T magnetic field.

Electrospray ionization. Proteins were used as received from Sigma Chemical (St. Louis, MO). Protein solutions with analyte concentrations of 3 to $10 \mathrm{pmol} / \mu \mathrm{L}$ in a 69:30:1 $\mathrm{MeOH}: \mathrm{H}_{2} \mathrm{O}: \mathrm{HOAc}$ matrix were pumped to the electrospray chamber through a 22-gauge Teflon tube at $4 \mu \mathrm{L} / \mathrm{min}$ with an Isco model SFC-500 microflow syringe pump. Optimum ion current was obtained with the electrospray syringe needle biased at $3.7 \mathrm{kV}$ and the desolvating capillary resistively heated to about $150^{\circ} \mathrm{C}$ by an applied current of $2.2 \mathrm{~A}$. The skimmer cone was biased at +5 to $+30 \mathrm{~V}$ to obtain desired electrospray ion velocities in the direction of the trapped ion cell.

FTICR detection. FTICR spectral performance was evaluated at both $5 \times 10^{-5}$ torr and $2 \times 10^{-7}$ torr pressures for a wide range of trapping and detection parameters. Trap potentials were independently optimized in the range 0 to $9.75 \mathrm{~V}$ for both the ion accumulation and detection events. The pulse sequence was initiated with an ion injection or beam event during which static trap potentials were maintained as ions accumulated in the cell. The accumulation potential was carefully chosen to match the kinetic energy of the ion beam, as will be discussed. A $9.75-\mathrm{V}$ potential applied to the source conductance limit after the injection event prevented additional ions from entering the cell during subsequent events. Following a variable delay of up to $1 \mathrm{~s}$ to allow for collisional cooling, the trap plate potentials were lowered, typically to $1 \mathrm{~V}$. Ions were excited to larger cyclotron orbits with either a linear excitation sweep from 0 to $100 \mathrm{kHz}$ at a sweep rate of $1000 \mathrm{~Hz} / \mu$ s or by a single on-resonance $100-\mu \mathrm{s}$ pulse; the magnitude of the excitation was systematically varied to optimize the FTICR signal magnitude for different experiment conditions. More spectra were acquired in direct mode over a $2.66-\mathrm{MHz}$ bandwidth with sufficient data points, typically $16 k$ to $32 k$, for the mass-to-charge ratio range of interest to obtain maximum mass resolution. Total experiment times were typically several hundred milliseconds and tran- sient reproducibility was sufficient to facilitate signal averaging to enhance spectral $S / N$ as desired. Mass resolution, mass accuracy, and relative mass magnitudes were obtained from mass spectra following a single zero fill, baseline correction, sine-bell apodization, and magnitude mode Fourier transform.

\section{Results and Discussion}

\section{Magnetic Field Focusing Electrospray Ionization}

The motivation for forming the electrospray in a radially homogeneous magnetic field is that the radial velocity component for ions dispersed from the electrospray needle or the skimmer nozzle is constrained to small cyclotron orbits. Even ions that initially leave the source with relatively large radial kinetic energies will assume orbits of only a few millimeters and hence should be accessible for FTICR detection. To demonstrate the importance of the magnetic field for delivering a large electrospray current to the mass analyzer, the dual cell was removed from the vacuum chamber and replaced by a probe-mounted Faraday cup initially positioned adjacent to the skimmer. An initial electrospray current of $370 \mathrm{pA}$ was measured for a $7.5-\mu \mathrm{M}$ solution of Gramicidin-5. As the probe was retracted, ion current was maintained at $90 \%$ of the initial current over the remaining $40-\mathrm{cm}$ length of the strong magnetic field. Rapid dissipation of the ion current as the Faraday cup reached the fringing magnetic field is indicative of electrospray beam divergence without some form of focusing. Actual electrospray ion current at the position in the magnet solenoid corresponding to the trapped ion cells was $350 \mathrm{pA}$. This value contrasts with current readings of about 1 pA that are obtained with early external source ESI/FTICR instruments [14-16]. Because the diameter of the Faraday cup is nearly twice that of the hole in the trap plate these measurements are only indicative of the total ion current reaching the cell. The magnitude of ion current entering the trapped ion cell is a function of the relative diameter of the ion beam and the alignment of the ion beam with respect to the hole in the trap plate. As will be shown, this greater than 100 -fold enhancement in ion current translates into a corresponding increase in spectral $S / N$.

Although placement of the electrospray source in the strong magnetic field overcomes the problem of inefficient ion injection, a new problem is created-an inaccessibility to the magnet bore for adequate differential pumping. With the external source design in which ESI occurs outside the magnet bore, an unlimited series of pumping stages permits any desired pressure differential to be achieved. However, the luxury of unlimited axial displacement is not available when the electrospray source is positioned in the magnet bore. Typical magnet bore lengths are 50 to $80 \mathrm{~cm}$, which suggests that at most 20 to $30 \mathrm{~cm}$ may separate the analyzer trapped ion cell and ESI source regions if 
both are to be positioned in the strong homogeneous region of the magnetic field. To achieve the necessary reduction in pressures, we devised the concentric tube network of differentially pumped vacuum chambers described in the experimental section and shown in Figure 1. Although pumping is by no means optimized, the potential of the design is evident as pressures are reduced over a $29-\mathrm{cm}$ distance from atrosphere at $1.5 \mathrm{~T}$ in the magnet bore to the $2 \times 10^{-7}$ torr range at $3.0 \mathrm{~T}$.

In an initial report on the magnetic field focusing interface [17], only ESI-FTICR spectra acquired in the high-pressure ( $5 \times 10^{-5}$ torr) trapped ion cell were shown; included was a spectrum of bovine albumin dimer with a mass of $132,532 \mathrm{Da}$. Pressure constraints limited mass resolution to between 70 and 100 in the mass range of interest. Spectra could not be obtained in the lower pressure cell because of alignment difficulties. Recently, however, the conductance limit between the cells was increased to $4.0 \mathrm{~mm}$ and the conductance limit adjacent to the skimmer assembly was reduced to $2 \mathrm{~mm}$. Overall system pressures remained the same, but electrospray ion current measured at $100 \mathrm{pA}$ was collected behind the analyzer cell. ESI spectra measured in the analyzer cell exhibited the expected improvement in mass resolution and for example, we have obtained mass resolution in excess of 4000 for cytochrome $c$. Attempts to significantly increase resolution by allowing ion thermalization delays of hundreds of seconds as performed by Henry and McLafferty [14-16] have been successful. In a slightly modified version of this ESI-FTICR interface a $200-\mu \mathrm{m}$ skimmer cone is utilized that allows operating pressures in the low $10^{-8}$ torr range. This modification has yielded resolving power over 20,000 for the $4+$ charge state of melittin. Presented in Figure 2 are comparison cytochrome $c$ spectra acquired at $5 \times 10^{-5}$ torr and $2 \times 10^{-7}$ torr, respectively. Signal magnitudes were improved by at least an order of magnitude compared to the high-pressure cell, despite the somewhat smaller total ion current. This is at least partially attributed to line shape reduction. The efficient acquisition of ESI/FTICR spectra at this reduced pressure has important implications with respect to efficient trapping and detection as will be discussed below.

\section{Ion Kinetic Energy and Trapping}

Two fundamentally different approaches are taken to trapping externally formed ions in the trapped ion cell. The first, in which trap plate potentials are gated below the kinetic energy of injected ions, has been used with several external source designs [25, 26] including the ESI/FTICR instrument of Henry and McLafferty et al. [14-16]. Although the trapping process is easily understood, disadvantages include a subsequent ejection of ions when trap potentials are reinstated [27] and a fundamental incompatibility with continuous sources such as electrospray, which re-
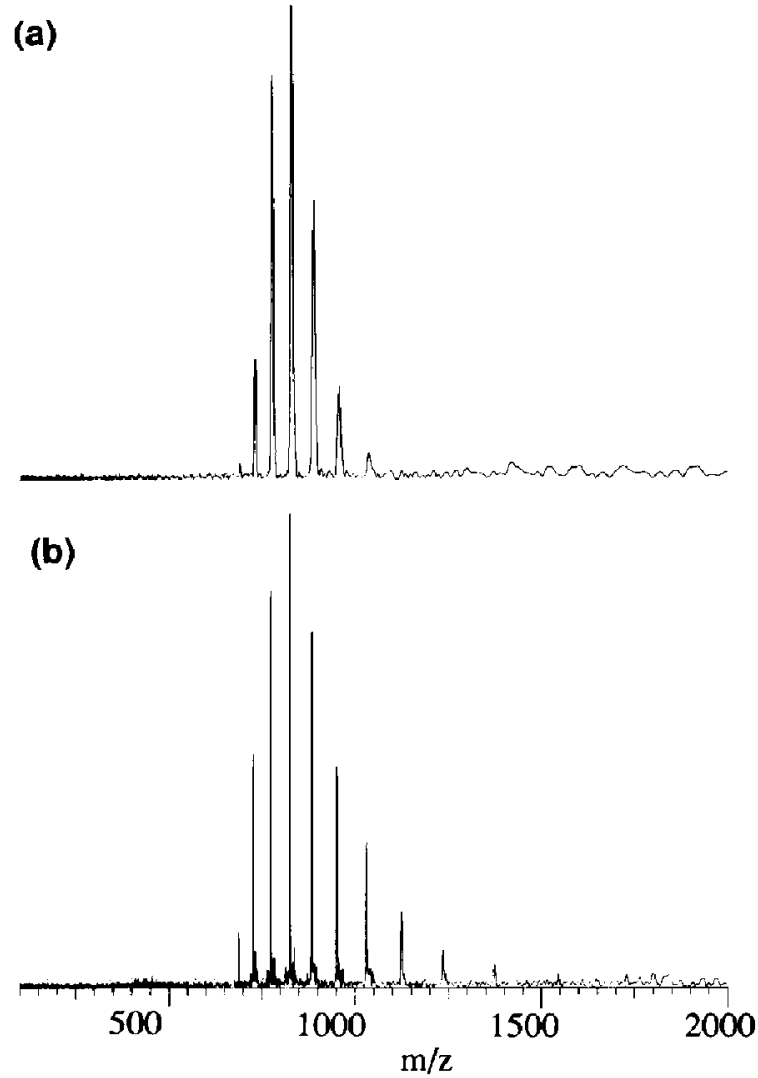

Figure 2. ESI/FTICR spectra acquired under pressure-limited conditions with (a) spectrum acquired at $5 \times 10^{-5}$ torr in the source cell and (b) spectrum acquired in the analyzer cell at $2 \times 10^{-7}$ torr. Mass resolution values are about 90 for the highpressure spectrum and about 1000 for the low-pressure spectrum

duces overall trapping efficiency. A second, more poorly understood approach is to maintain static trap potentials as ions bombard the cell and accumulate in the potential well between the trap plates $[28,29]$. The advantage of accumulated trapping for electrospray ionization is that, provided an efficient trapping mecharism is found, ionization duty cycle is superior and FTICR sensitivity should also improve.

We have found that appropriate conditions for efficient trapping of externally generated electrospray ions can be achieved by carefully matching the z-axis kinetic energy of the ion beam with the trap plate potential. The most important factor contributing to the kinetic energy of the ion beam is the distance between the desolvating capillary and the skimmer cone. Energies approach several hundred electronvolts for very small capillary-skimmer distances, presumably due in part to field penetration of the capillary, but are on the order of tens of electronvolts or less at the selected distance of $0.45 \mathrm{~cm}$. The distance is also critical to the pressure achieved in the analyzer trapped ion cell, but fortunately pressure minima occur at 
capillary-skimmer distances that correspond to kinetic energies of only a few electronvolts.

With the skimmer-capillary distance positioned for lowest cell pressure, typically $4.5 \mathrm{~mm}$, the skimmercone potential then becomes the controlling parameter for the ion kinetic energy. Retarding grid studies performed at the cell indicate that electrospray ions achieve a kinetic energy equal to approximately one third of the potential drop between the skimmer and first conductance limit, which is at ground potential. Coincidentally, the trap potential necessary to accumulate electrospray ions is found to mimic the kinetic energy profile generated with the retarding grid measurement. This is illustrated in Figure 3 for horse myoglobin ions. A retarding grid measurement in Figure $3 a$ yields a derivative plot in Figure $3 \mathrm{c}$ indicative of ion kinetic energy. The stacked plot of electrospray FTICR

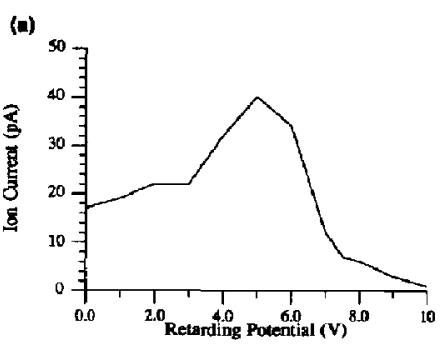

(b)

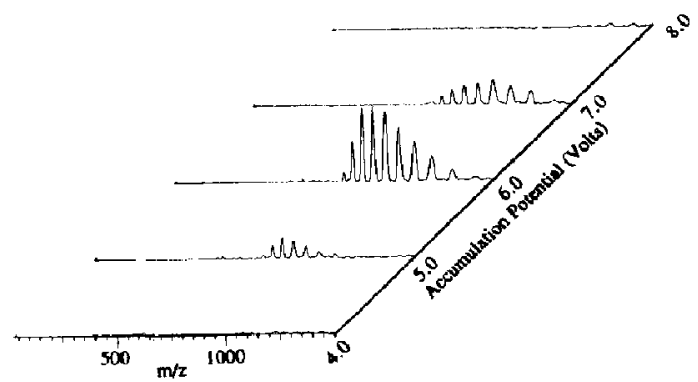

(c)

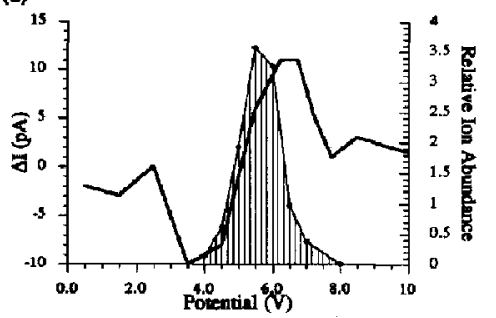

Figure 3. (a) Retarding grid profile of electrospray ion current for a $10 \mathrm{pmol} / \mu \mathrm{L}$ solution of horse myoglobin (MW $=16950.7)$. The source trap plate served as the retarding grid and the ion current was measured on the electrode separating source and analyzer cells. (b) Stacked plots of horse myoglobin ESI/FTICR spectra acquired as a function of increasing trap potentials. (c) Overlay of derivative plot of retarding grid profile (bold line) onto the profile of relative peak maxima from ESI/FTICR spectra acquired at increasing trap potential. spectra acquired at increasing trap potential in Figure $3 b$ yields the profile in Figure $3 c$ of FTICR signal magnitude as a function of trap potential. The close overlap of profiles indicates that the trapping mechanism used to acquire FTICR spectra is kinetic energy dependent and that variable trap potential studies can be an effective tool for measuring ion kinetic energies of externally generated ions.

Figure 4 demonstrates the ability to tune the skimmer potential to acquire electrospray ions at any desired trap potential. It is noteworthy that a narrower energy distribution is observed at lower skimmer potentials. For example, in the 5.0-V skimmer case ions are successfully trapped only over a $2.5-\mathrm{V}$ range, while a 15.0-V skimmer cone potential permits ion trapping over a $5.0-\mathrm{V}$ range. This kinetic energy broadening is due to a convolution of two factors that influence the kinetic energy of ions leaving the skimmer cone. The first of these factors is related to the kinetic energy imparted to the ions from the supersonic expansion and is a function of the Mach number of supersonic expansion, which defines the particle velocity and the mass of the particle $[30,31]$. In addition, because the skimmer cone is biased and the particles passing through it are charged, the particles are subject to an additional force that is proportional to the number of charges and to the magnitude of the electric field. In this apparatus the characteristics of the supersonic expansion are relatively invariant and the resulting kinetic energy contribution is constant. Due to the relatively low Mach number of the expansion and the fact that a grounded skimmer cone produces little or no ion current at the cell, it appears that this contribution to ion kinetic energy is insignificant. Thus, by manipulating the potential applied to the skimmer cone, fine control of the kinetic energy of the ion population is achieved.

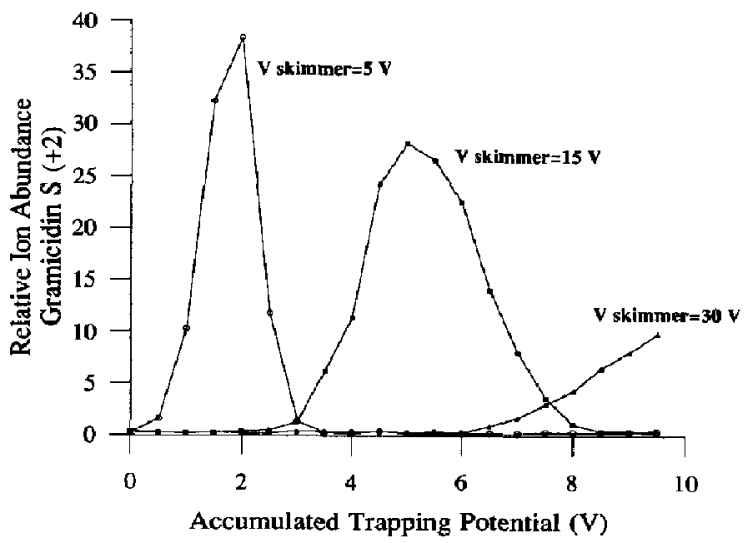

Figure 4. Accumulated trapping profiles at various skimmer cone potentials. The profiles correspond to a skimmer cone bias of $\bigcirc 5 \mathrm{~V}, \square 15 \mathrm{~V}$, and $\Delta 30 \mathrm{~V}$. In general, ions are most efficiently accumulated with trap potentials at approximately one third of the skimmer cone potential when the skimmer-capillary distance is optimized for low analyzer cell pressure. 
The question of a trapping mechanism by which these electrospray ions are retained is now considered. From the data in Figure $3 b$ we may argue for a differential trapping process in which ions are trapped for detection if they are within a narrow energy range that allows penetration of the trapping barrier but have insufficient kinetic energy for subsequent expulsion. Thus, for example, the energy difference defined in Figure $3 c$ between retarding grid profile and the trap potential profile corresponds to this range of ion energies that facilitates trapping. Measurement of this energy differential was not precise in the experiment because of insufficient stability, but suggests that only ions with roughly as much as a few tenths of an electronvolt of excess kinetic energy upon penetrating the trap will be retained.

In evaluating the actual mechanism by which ions lose this few tenths of an electronvolt and are trapped, we can make an argument that collisional damping is important given the relatively high pressures at which ions are injected. However, from the FTICR ion magnitude profiles plotted as a function of increasing ion injection period in Figure $5 \mathrm{a}$ a collisional trapping mechanism may be disputed. Profiles for cytochrome $c$ acquired at $5 \times 10^{-5}$ and $2 \times 10^{-7}$ torr exhibit similar rates for ion trapping, a feature that contradicts a simple collisional damping process. Alternatively, we believe that an additional electric field mechanism is responsible for redirecting axial ion kinetic energy such that because of incompatibility in the phase relationship between trapping and magnetron motion, an ion cannot leave the cell prior to detection.

The arguments provided above against collisional trapping process do not imply that collisional cooling is not important to the detection of electrospray ions. The contrast between the linear high-pressure profiles for cytochrome $c$ and bovine albumin in Figure 5a and the sigmoidal shape to the low-pressure cytochrome $c$ profile suggests the importance of collisional stabilization of the injected ion cloud prior to detection. Also shown in Figure $5 \mathrm{a}$ is a cytochrome $c$ profile generated for the same injection periods as for the sigmoidal curve but with a brief delay period added. The additional cooling period is sufficient for stabilization to detect a larger number of injected ions. The data are in agreement with the observation of Henry and McLafferty et al. [16] that very long collisional delays (as long as $1000 \mathrm{~s}$ ) are necessary at $10^{-9}$ torr pressure to achieve the best signal intensity. Pulsed valves should provide an effective altemative that minimizes experiment time.

Another feature of interest in the ion accumulation profiles shown in Figure $5 \mathrm{a}$ is that for typical protein samples with analytical concentrations of 5-10 pmol $/ \mu \mathbf{L}$, the optimum filling time is dependent on the charge state and charge distribution of the ion population. For example, the cytochrome $c$ spectra to which Figure 5a refers contain a charge envelope from $16+$ to $12+$ while the bovine albumin spectra demonstrate a charge envelope from $56+$ to $32+$. Thus in
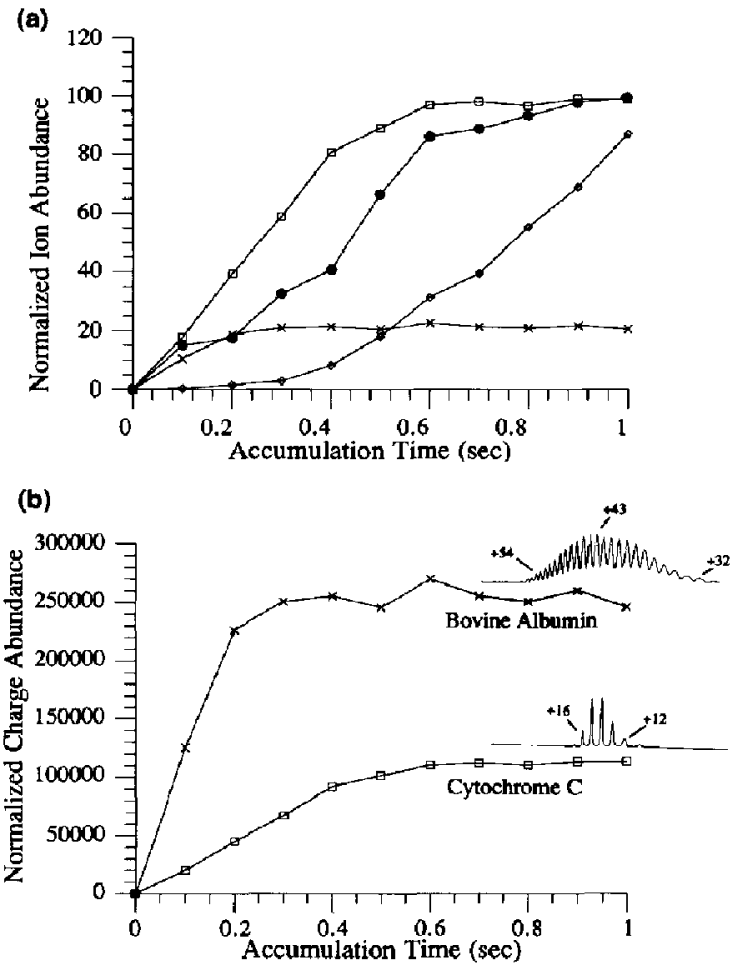

Figure 5. Profiles of ESI /FTICR signal magnitude acquired as a function of increasing ion accumulation time. (a) Relative abundance as measured by base peak magnitude for $\square$ cytochrome $c$ at $2 \times 10^{-5}$ torr $\times$ bovine albumin at $2 \times 10$ torr: $\diamond$ cytochrome $c$ at $2 \times 10^{-7}$ torr; cytochrome $c$ at $2 \times 10^{-7}$ torr with collisional cooling time equal to $1,0 \mathrm{~s}$ accumulation time. (b) Normalized charge abundance as measured from summation of products of signal magnitudes and charge state for $\square$ cytochrome $c$ and $x$ bovine albumin.

the case of multiply charged ions in the trapped cell there is a clear distinction between charge capacity and ion capacity. The charge capacity of the trapped ion cell is a function of the geometry of the cell, the magnetic field strength, and the applied trapping potentials. The ion capacity of the cell is a function of the charge capacity of the cell and the charge state of the ion of interest. Consequently, the trapped ion cell has a smaller ion capacity for highly charged ions than for singly charged ions. However, as Figure 5b suggests, the charge capacity appears to be greater for ion populations composed of large densely charged ions as opposed to smaller, moderately charged ions. This phenomenon may prove to be advantageous for large highly charged ions in which a single ion could carry ample charge sites to produce a detectable image current in the absence of space charge perturbation of the electric field.

\section{Excitation and Detection Parameters}

With a reliable method established to reproducibly inject and trap large numbers of electrosprayed ions, 
studies were next undertaken to determine optimum FTICR excitation and detection parameters for these large, highly charged ions. The selection of optimum broadband swept excitation parameters, including bandwidth and sweep rate, was found to depend strongly on the analyte mass, mass-to-charge ratio, and background pressure. For example, the swept excitation profiles presented in Figure 6 for equine myoglobin indicate a discrimination against the more highly charged ions as slower, higher energy sweeps are employed. This trend is more pronounced at high pressure but still occurs at $2 \times 10^{-7}$ torr. It was also observed that the optimum sweep rate varied inversely with the mass of the protein. Profiles of singlefrequency excitation pulses at increasing energy were then obtained for specific charge states of cytochrome $c$ and are shown in Figure 7 for the two pressure regimes. Both sets of profiles exhibit decidedly nonideal behavior in which ions with fewer charges are favored at higher excitation voltages. This observation is consistent with the swept excitation data in Figure 6. Although the charge discrimination is still observed in the profiles at $2 \times 10^{-7}$ torr, the excitation maxima are shifted toward higher energies and, consequently, improved FTICR performance.

A probable explanation for the mass and charge discrimination described above and shown in Figures 6 and 7 is that for a hard-sphere collision model, larger mass ions and more highly charged ions will experience an increased number of collisions. For example, the hard-sphere model predicts that collision frequency scales with velocity and therefore inversely with mass-to-charge ratio. Bovine albumin dimer is about 100 times heavier than Gramicidin-S and conse-

(a)

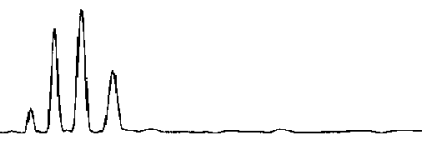

(b)

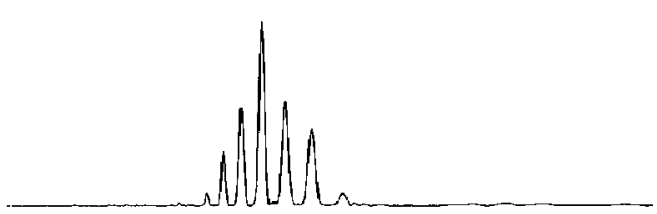

(c)

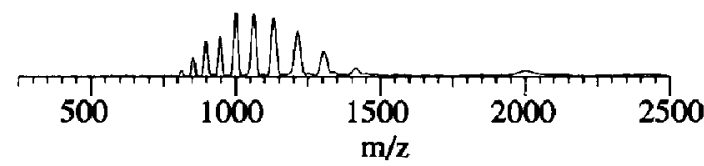

Figure 6. ESI/FTICR spectra for horse myoglobin acquired at $5 \times 10^{-5}$ torr with sweep rates of (a) $500 \mathrm{~Hz} / \mu \mathrm{s}$, (b) 1000 $\mathrm{Hz} / \mu \mathrm{s}$, (c) $2000 \mathrm{~Hz} / \mu \mathrm{s}$, and otherwise identical conditions.

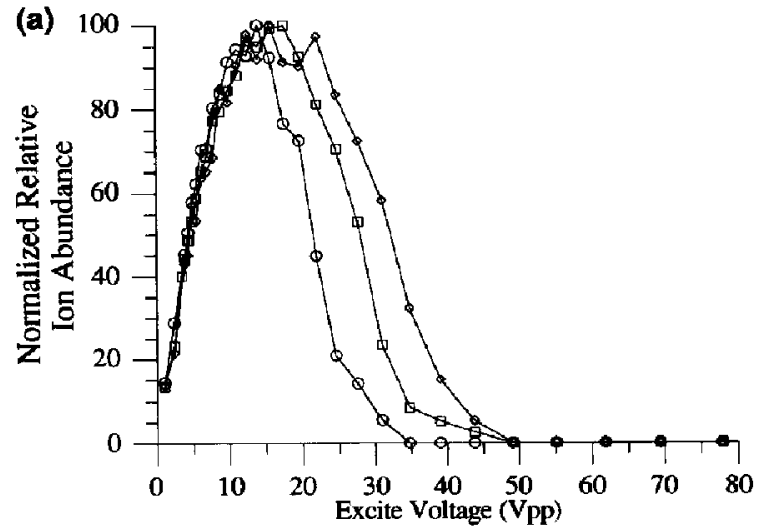

(b)

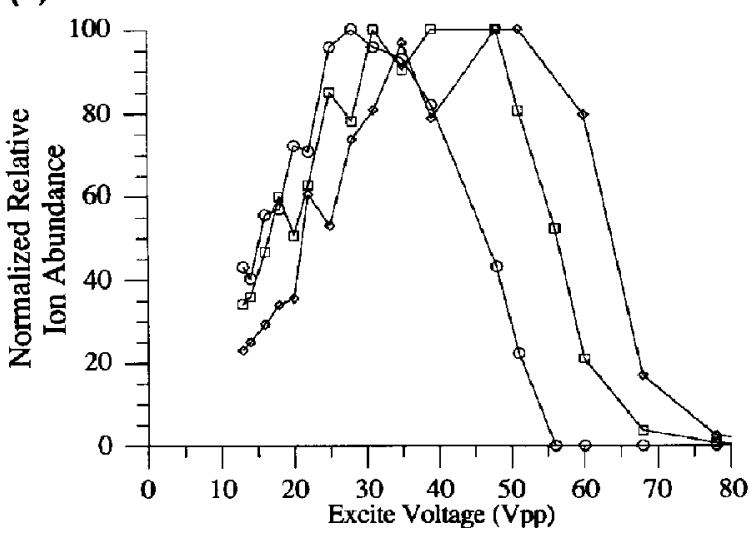

Figure 7, Profiles of cytochrome $c$ signal magnitude from ESI/FTTCR spertra following $100-\mu$ s single-frequency excitation at increasing excitation voltage: (a) excitation profiles at $4 \times 10^{-5}$ torr for $O+18 ; \square+15 ; 0+13$ charge states and (b) excitation profiles acquired at $2 \times 10^{-7}$ torr for $O+18 ; \square+14 ; \diamond+12$ charge states.

quently has a significantly larger collisional cross section. The reduction in FTICR signal magnitude with increasing charge in the single frequency excitation profiles in Figure 7 is also consistent with an increase in collision frequency. For isomass ions, the velocity increases directly with increasing charge state, which suggests for example that the $18+$ charge ion is traveling at about 2.25 times the velocity of the $8+$ charge ion and consequently should experience a 2.25 -fold increase in collision frequency. Thus the usual condition in FTICR that ions of only slightly different mass and identical charge will exhibit mass resolution values that scale inversely with mass does not hold true for electrospray ions. This is indicated with the data presented in Figure 8 for the cytochrome $c$ spectrum acquired under pressure-limited conditions in Figure $2 \mathrm{~b}$. In this example the best mass resolution $x$ massto-charge ratio product is obtained for low charge state, high mass-to-charge ratio ions that undergo the fewest collisions. This distinctive aspect of ESI/FTICR spectra should be observed even at low pressures if 


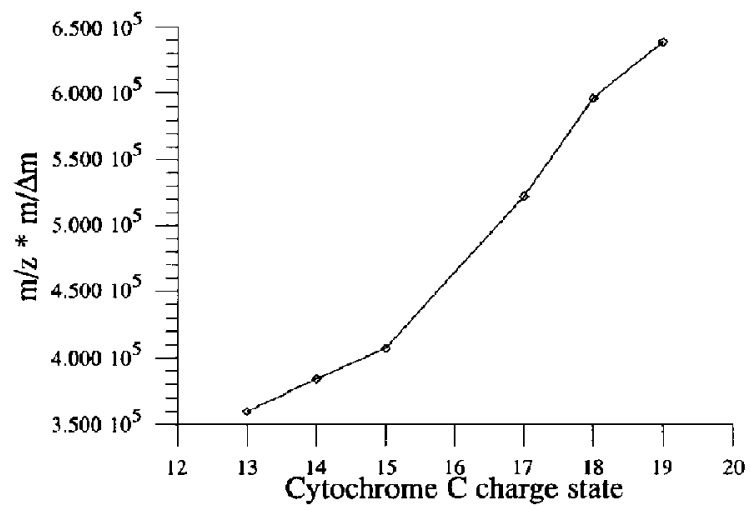

Figure 8. Product of mass-to-charge ratio and mass resolution for individual charge states in the ESI/FTICR spectrum of cytochrome $c$ acquired at $2 \times 10^{-7}$ torr.

collisional relaxation is the primary signal damping mechanism.

One final interesting feature of the data acquisition parameters for electrospray ions is that the optimum trapping potential for trapping electrospray ions is not the best trap potential for detecting these ions. The data in Figure 9 are FTICR signal magnitude values for cytochrome $c$ ions acquired at $5.05 \mathrm{~V}$ but detected at trap potentials ranging from 0 to $10 \mathrm{~V}$. In general, signal amplitude is highest at surprisingly low trapping potential given the relatively high kinetic energy with which the ions were trapped. The data suggest that the dominant collision-based relaxation process at high pressures is in the radial rather than axial dimension. This is because collisional redistribution of ion kinetic energy into the axial dimension cannot be an effective relaxation process for these very large ions, because ion magnitude would be expected to decrease with reduced trap potentials. Instead, the relaxation

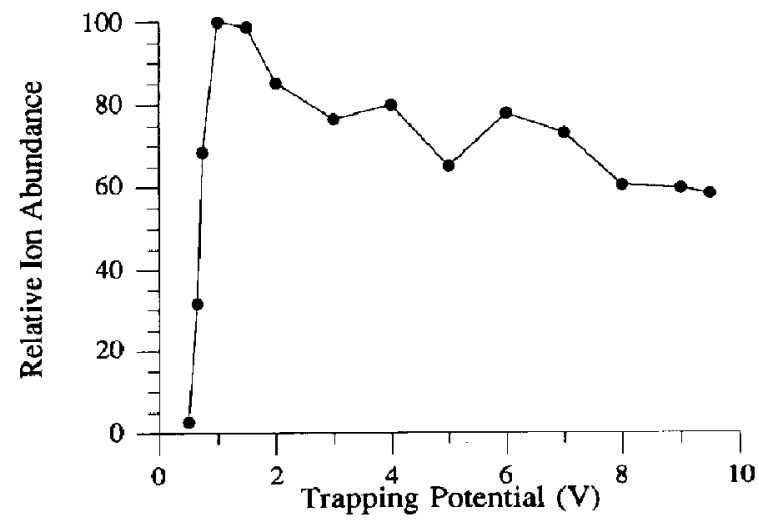

Figure 9. ESI/FTICR base peak magnitude from cytochrome $c$ spectra acquired at increasing trap potential. Analyzer pressure was $2 \times 10^{-7}$ torr, ion injection occurred at $5.05 \mathrm{~V}$, and the ion accumulation period was $800 \mathrm{~ms}$. process at high pressures is probably related to dispersion of the ion cloud in the radial dimension because a reduction in the radial trapping electric field reduces this effect. McIver has described this form of ion loss, which is based upon a random walk of ions in the strong $\mathrm{E} \times \mathrm{B}$ field at the cell perimeter [32]. At the pressures used for detection this process apparently competes with and can defeat efforts to perform McLafferty's multiple reexcitation experiment [33], which requires that collisional cooling of the cyclotron radius dominate.

\section{Acknowledgments}

This work is supported by the Welch Foundation (F-1138), the Amold and Mabel Beckman Foundation, the Texas Advanced Research Program, and the National Science Foundation (CHE9013384 and CHE9057097).

\section{References}

1. Fenn, J. B.; Mann, M.; Meng, C. K.; Wong, S. F; Whitehouse, C. M. Mass Spectrom. Rev. 1990, 9, 37-70.

2. Smith, R. D.; Loo, J. A.; Edmonds, C. C.; Barinaga, C. J.; Udseth, H. R. J. Chromatography 1990, 516, 157-165.

3. Feng, R.; Bouthiller, F.; Konishi, Y.; Cygler, M. Proceedings of the 39th ASMS Conference on Mass Spectrometry and Allied Topics, Nashville, TN, 1991; pp 1159-1160.

4. Van Berkel, G. J.; McLuckey. S. A.; Glish, G. L. Proceedings of the 38th ASMS Conference on Mass Spectrometry and Allied Topics, Tucson, AZ, 1990; $\mathrm{pP}$ 12-13.

5. Wong, S. F.; Meng, C. K.; Fenn, J. B. J. Phys. Chem. 1988, 92, 546-550.

6. Meng, C. K.; Larsen, B. S.; McEwen, C. N. J. Rapid Commun. Mass Spectrom. 1990, 4, 151-155.

7. Aleksandrov, M. L.; Baram, G. I.; Gall, L. N.; Krasnov, V. N.; Kusner, Y. S.; Mirgorodskaya, O. A.; Nikolaev, V. I.; Shkurov, V. A. Bioorg. Khim. 1985, 11, 700-705.

8. Aleksandrov, M. L.; Besuklandikov, P. V.; Grachev, M. A.; Elyakova, L. A; Zyyaginsteva, T. N.; Kondratsev, V. M.; Kusner, Y. S.; Mirgorodskaya, O. A.; Fridlyansky, G. V. Bioorg. Khim. 1986, 12, 1689-1692.

9. Bateman, R. H.; Major, H. J.; Woolfit, A. R. Presented at the 12th International Mass Spectrometry Conference, Amsterdam, The Netherlands, August 26-30, 1991.

10. Allen, M. H.; Lewis, I. A. S. I. Rapid Commun. Mass SpecErom. 1989, 4, 255-258.

11. Wanczek, K. P. Int. J. Mass Spectrom. Ion Proc, 1989, 95, $1-38$.

12. Wang, M.; Marshall, A. G. Anal. Chem. 1990, 62, 515-520.

13. Laude, D. A. Jr.; Johlman, C. L,; Brown, R. S.; Weil, A.; Wilkins, C. L. Mass Spectrom. Rev. 1986, 5, 107-119.

14. Henry, K. D.; McLafferty, F. W. Org. Mass Spectrom. 1990 , $25,490-492$

15. Henry, K. D.; Williams, E. R.; Wang, B. H.; McLafferty, F. W.; Shabanowitz, J.; Hunt, D. W. Proc. Natl. Acad. Sci. U.S.A. 1989, 86, 9075-9078.

16. Henry, K. D.; Quirn, J. P.; McLafferty, F. W. J. Am. Chem. Soc. 1991, 113, 5447-5449.

17. Hofstadler, S. A.; Laude, D. A. Jr. Anal. Chem., 1992, 64, 569-572.

18. Hamdan, M.; Curcuto O. Int. J. Mass Spectrom. Ion Proc. 1991, $108,93-113$. 
19. Lebrilla, C. B.; Amster, I. J.; Mclver, R. T. Int. I. Mass Spectrom. Ion Proc. 1989, 87, R7-R13.

20. Kofel, P.; Alleman, M.; Kellerhals, H.; Wanczek, K. P. Int. J. Mass Spec. Ion Proc. 1989, 87, 237-247.

21. Ijames, C. F.; Markey, S. P. Proceedings of the 39th ASMS Conference on Mass Spectrometry and Allied Topics, Nashville, TN, 1991; pp 1548-1549.

22. Kerley, E. L.; Buchanan, M. V.; Cook, K. D.; Shangholi, M. Proceedings of the 39th ASMS Conference on Mass Spectrometry and Allied Topics, Nashville, TN, 1991; pp 1175-1176.

23. Katta, V.; Chowdhury, S. K.; Chait, B. T. J. Am. Chem. Soc. $1990,112,5348-5349$.

24. Riegner, D. E.; Hofstadler, S. A.; Laude, D. A. Jr. Anal. Chem. 1991, 63, 261-268.

25. Marruyana, S.; Anderson, L. R.; Smalley, R. E. Rev. Sci. Instrum. 1990, 61, 3686-3693.
26. Alleman, M.; Kofel, P.; Kellerhals, H.; Wanczek, K. P. Int. J. Mass Spec. Ion Proc. 1987, 75, 47-54.

27. Hofstadler, S. A.; Laude, D. A. Jr. Int. I. Mass Spectrom. Ion Proc. 1990, 101, 65-78.

28. Beu, S. C.; Laude, D. A. Jr. Int. J. Mass Spectrom Ion Proc., in press.

29. Allison, J.; Stepnowski, R. M. Anal. Chem. 1987, 59, 1072A-1088A.

30. Miller, D. R. In Atomic and Molecular Beam Methods; G. Scoles, Ed.; Oxford, 1988; pp 2-53.

31. Kantrowitz, A.; Grey. J. Rev. Sci. Instrum. 1951, 22, 328-337.

32. Francl, T. J.; Fukuda, E. K.; McIver, R. T. Jr. Int. J. Mass Spec. Ion Phys. 1983, 50, 151-167.

33. Williams, E. R.; Henry, K. D.; McLafferty, F. W. I. Am. Chem. Soc. 1990, 112, 6157-6162. 